\title{
Immunometabolic Determinants of Chemoradiotherapy Response and Survival in Head and Neck Squamous Cell Carcinoma
}

Rosemarie Krupar, ${ }^{* \dagger}$ Matthias G. Hautmann, ${ }^{\ddagger}$ Ravi R. Pathak, ${ }^{\dagger}$ Indu Varier, ${ }^{\S}$ Cassandra McLaren, ${ }^{\dagger}$ Doris Gaag, Claus Hellerbrand, Matthias Evert, ${ }^{\top}$ Simon Laban, ${ }^{* *}$ Christian Idel, ${ }^{* \dagger}$ Vlad Sandulache, ${ }^{\dagger}$ Sven Perner, ${ }^{*}$ Anja K. Bosserhoff, and Andrew G. Sikora ${ }^{\dagger}$

From Pathology of the University Medical Center Schleswig-Holstein,* Campus Lübeck and Research Center Borstel, Leibniz Center for Medicine and Biosciences, Lübeck, Germany; the Department of Radiotherapy, ${ }^{\ddagger}$ University Hospital Regensburg, Regensburg, Germany; the Department of Otolaryngology-Head and Neck Surgery, ${ }^{\dagger}$ Baylor College of Medicine, Houston, Texas; the Department of Pediatrics, ${ }^{\S}$ Tulane University, New Orleans, Louisiana; the Institute of Pathology, "University of Regensburg, Regensburg, Germany; the Department of Oto-Rhino-Laryngology, Head and Neck Surgery, ** University Medical Center, Ulm, Germany; the Department of Otorhinolaryngology, ${ }^{\dagger \dagger}$ University Hospital Schleswig-Holstein, Lübeck, Germany; and the Institute of Biochemistry, "Friedrich-Alexander University Erlangen-Nürnberg, Erlangen, Germany

Accepted for publication September 21, 2017.

Address correspondence to Rosemarie Krupar, M.D., Pathology of the University Medical Center Schleswig-Holstein Campus Lübeck and Research Center Borstel, Leibniz Center for Medicine and Biosciences, Ratzeburger Allee 160 (Building 50), 23538 Lübeck, Germany. E-mail: rosemarie. krupar@uksh.de.

\begin{abstract}
Tumor immune microenvironment and tumor metabolism are major determinants of chemoradiotherapy response. The interdependency and prognostic significance of specific immune and metabolic phenotypes in head and neck squamous cell carcinoma (HNSCC) were assessed and changes in reactive oxygen species were evaluated as a mechanism of treatment response in tumor spheroid/immunocyte co-cultures. Pretreatment tumor biopsies were immunohistochemically characterized in $73 \mathrm{HNSCC}$ patients treated by definitive chemoradiotherapy and correlated with survival. The prognostic significance of CD8A, GLUT1, and COX5B gene expression was analyzed within The Cancer Genome Atlas database. HNSCC spheroids were co-cultured in vitro with peripheral blood mononuclear cells (PBMCs) in the presence of the glycolysis inhibitor 2-deoxyglucose and radiation treatment followed by PBMC chemotaxis determination via fluorescence microscopy. In the chemoradiotherapy-treated HNSCC cohort, mitochondrial-rich (COX5B) metabolism correlated with increased and glucose-dependent (GLUT1) metabolism with decreased intratumoral CD8/CD4 ratios. High CD8/CD4, together with mitochondrialrich or glucose-independent metabolism, was associated with improved short-term survival. The Cancer Genome Atlas analysis confirmed that patients with a favorable immune and metabolic gene signature (high $C D 8 A$, high COX5B, low GLUT1) had improved short- and long-term survival. In vitro, 2-deoxyglucose and radiation synergistically up-regulated reactive oxygen species-dependent PBMC chemotaxis to HNSCC spheroids. These results suggest that glucose-independent tumor metabolism is associated with CD8-dominant antitumor immune infiltrate, and together, these contribute to improved chemoradiotherapy response in HNSCC. (Am J Pathol 2018, 188: 72-83; https://doi.org/10.1016/j.ajpath.2017.09.013)
\end{abstract}

Head and neck squamous cell carcinoma (HNSCC) is the fourth most common cancer in the United States with almost 62,000 new cases per year. ${ }^{1}$ Although either single-modality surgery or radiotherapy is often used for early (stage I to II) disease, chemoradiotherapy (CRT) is frequently used for tumors presenting at more advanced stages. ${ }^{2}$ The success of CRT treatment is influenced by diverse factors including anatomic location and stage, etiology due to infection with the human papillomavirus (HPV), pathologic features, genomic and epigenetic changes in the tumor, prior therapy, and other factors. As is the case for many solid tumors, the tumor microenvironment is a significant determinant of CRT response, particularly the tumor immune microenvironment (TIME) and the tumor metabolic status.,

Supported by the German Cancer Aid (R.K.), the German Research Foundation (KFO262) grant 190230491 (R.K.), and the Foundation Tumor Research Head and Neck (Stiftung Tumorforschung Kopf Hals) (R.K.).

Disclosures: S.L. is an advisory board member for AstraZeneca. 
Solid markers to define the tumor microenvironment (TME) according to CRT response are, however, still missing.

Tumor oxygenation and functional mitochondria are crucial for successful CRT response. ${ }^{5,6}$ Mitochondria, as the most important producers of reactive oxygen species (ROS), are the endmediators of CRT-induced cell damage. Apoptosis is initiated via ROS signaling, and ROS also induce damage to mitochondrial DNA. Additionally, ROS are necessary for fixing radiationinduced DNA strand breaks. ${ }^{7-9}$ The hallmark of radiation resistance, on the other hand, is hypoxia and increased use of glycolysis and production of lactate, even in the presence of oxygen (aerobic glycolysis/Warburg effect) for energy production instead of more effective, mitochondrial oxidative phosphorylation. ${ }^{10,11}$ Aerobic glycolysis is observed independently of tumor oxygenation and has been shown to follow a compartmentalized spatial distribution in the TME, leading simultaneously to areas with predominantly oxidative phosphorylation and predominantly glycolytic areas, which coexist in a mutual symbiosis. ${ }^{12}$ Nevertheless, solid tumors in general, and HNSCC in particular, are characterized by an accumulation of lactate contributing to radioresistance by scavenging of ROS. ${ }^{13}$

Additionally, tumor cell-derived lactate is known to inhibit $\mathrm{CD}^{+}$cytotoxic T cells (CTL). ${ }^{14,15}$ A CTL-dominant tumor immune infiltrate, however, has been shown in many different solid tumors to be predictive of therapy response. In HPVpositive oropharyngeal squamous cell carcinomas (OPSCC), which are known for favorable response to CRT, we have already shown an increased CD8/CD4 ratio as well as a correlation of a CTL-dominant tumor immune infiltrate with a mitochondrial-rich tumor metabolism. ${ }^{16}$

Therefore, it is hypothesized that a glucose-dependent or mitochondrial-rich tumor metabolism can determine whether immunosuppression or effective antitumor immune response predominates in the TIME. It is specifically anticipated that tumors characterized by both a CTL-dominant tumor immune infiltrate and a mitochondrial-rich tumor metabolism would experience the most favorable response to CRT.

To elucidate the interdependencies of tumor metabolism and the TIME, and their role in therapy response, immune and metabolic characteristics as well as outcomes of HNSCC treated with primary CRT were correlated in both an institutional cohort and in The Cancer Genome Atlas (TCGA) HNSCC cohort. Additionally, dependency of radiation effects on ROS and metabolic modulation in vitro was examined in a three-dimensional HNSCC spheroid/ immune cell co-culture model to determine whether ROS production may link metabolic status to immune response.

\section{Materials and Methods}

\section{Patient Data and Tumor Material}

A retrospective cohort of 73 HNSCC patients, receiving CRT in definitive indication at the Department of Radiotherapy at the University Hospital Regensburg from 2005 to 2010, was established in cooperation with the Institute of Pathology of the University of Regensburg. Overall survival data were obtained by the cancer registry of the Tumor Center Regensburg. All patients received nonsurgical treatment consisting of cisplatinbased CRT. Patient characteristics and social histories were obtained from medical reports. Representative formalin-fixed, paraffin-embedded tumor tissues were retrieved from the archives of the Institute of Pathology of the University of Regensburg. The study was approved by the institutional review board of the University of Regensburg.

\section{Immunohistochemistry}

Immunostaining was performed on 4- $\mu$ m-thick sections of formalin-fixed, paraffin-embedded specimen after deparaffinization and microwave-based antigen retrieval as previously described. ${ }^{16}$ The IView DAB Detection Kit (Roche, Basel, Switzerland) was used on a Ventana BenchMark platform (Roche) for the following antibodies: CD4 (Clone SP35; Roche), CD8 (Clone SP57; Roche), forkhead box P3 (Clone 236A/E7; Thermo Fisher Scientific, Bremen, Germany), and cytochrome c oxidase subunit 5B (COX5B) (Clone 1E8; Sigma-Aldrich, Taufkirchen, Germany). Immunostaining for glucose transporter 1 (GLUT1) (Clone SPM498; LabVision, Fremont, CA) was performed manually with an appropriate horseradish peroxidase-conjugated secondary antibody.

\section{Evaluation of Immunohistochemical Staining}

Whole slides were used for analysis of immunohistochemistry. Tumor infiltrating lymphocytes were manually counted within tumor cell nests of four to five representative high power fields and an average number of lymphocytes per high power field was calculated.

The expression levels of COX5B and GLUT1 by tumor cells were semiquantitatively evaluated using the $\mathrm{H}$-score. Here, the percentage of strongly stained cells $(3 \times)$, the percentage of moderately stained cells $(2 \times)$, the percentage of weakly stained cells $(1 \times)$, and the percentage of unstained cells $(0 \times)$ were added, generating a score between 0 and 300 . All immunohistochemical evaluations were performed by an experienced pathologist blinded to patient outcome.

\section{TCGA Database Analysis}

TCGA data bank analysis was performed on the CBioPortal for Cancer Genomics website (http://www.cbioportal.org, last accessed August 2016). The Head and Neck Squamous Cell Carcinoma Provisional TCGA cohort with available mRNA data was analyzed for mRNA up- or down-regulation stratified according to mean mRNA expression levels for all tumors (zscore threshold $=0$ ). mRNA and clinical data were downloaded for further SPSS software-based survival analyses (SPSS software version 22.0; IBM SPSS Statistics, Armonk, NY).

\section{Cell Line and Tumor Spheroids}

HPV16-positive UM-SCC-47 cells ${ }^{17}$ were maintained in a combination of Dulbecco's modified Eagle's medium, 10\% fetal calf serum, and penicillin-streptomycin, $1 \%$. 
Spheroids were created by the hanging drop method. Briefly, 20,000 UM-SCC-47 cells were suspended in $20 \mu \mathrm{L}$ of complete medium and placed on the lid of a 24-well plate. After 4 to 5 days, hanging drops were transferred into agarose-coated wells.

\section{Radiation and Clonogenic Assay}

Cell irradiation was performed with the RS 2000 Biological X-ray Irradiator (Rad Source, Buford, GA). UM-SCC-47 cells $(n=500$ to 1000) were seeded in 6-well plates and exposed to different single radiation doses after sufficient adhesion time (4 to 24 hours). Fourteen days later, colonies were fixed with $10 \%$ formalin and stained with hematoxylin for 10 minutes. Colonies (cell aggregations with $>50$ cells) were counted, and the surviving fraction was calculated by dividing the number of colonies formed by the number of cells plated, multiplied by the plating efficiency.

\section{Spheroid Immune Cell Co-Cultures}

Peripheral blood mononuclear cells (PBMCs) were collected from fresh heparinized blood of healthy donors and processed immediately. After Ficoll-Hypaque density gradient centrifugation and carboxyfluorescein succinimidyl ester (CFSE)-labeling according to the manufacturer's instructions (CellTrace CFSE Cell Proliferation Kit, Life Technologies, Gaithersburg, MD), PBMCs were added to spheroids in agarose-coated wells of 24-well plates.

Migration of CFSE-labeled PBMCs toward tumor spheroids was monitored with an inverted microscope by recording immune cell attraction around spheroids at $\times 10$ magnification. The number of fluorescent PBMCs aggregating around one spheroid was determined by ImageJ software version 1.49v (NIH, Bethesda, MD; http://imagej. nih.gov/ij) (Supplemental Figure S1).

\section{ROS Measurement}

UM-SCC-47 $\left(2.5 \times 10^{5}\right.$ to $\left.5 \times 10^{5}\right)$ were seeded in a T25 culture flask and incubated for 4 days. ROS production was measured the day after treatment. Cells were trypsinized, and $200 \mu \mathrm{L}$ of $10 \mu \mathrm{mol} / \mathrm{L} \mathrm{2} 2^{\prime}, 7^{\prime}$-dichlorofluorescin diacetate $(\mathrm{CM}$ H2DCFDA; Molecular Probes, Eugene, OR) containing phosphate-buffered saline with $\mathrm{Ca}++$ and $\mathrm{Mg}++$ was added to each well. After incubation at $37^{\circ} \mathrm{C}$ for 30 minutes and respective treatment, ROS-producing cells were captured by a fluorescence microscope, and fluorescence intensity was quantified by ImageJ software.

\section{Statistical Analysis}

Unpaired two-tailed $t$-test was applied for discrimination of mean CD8/CD4 ratios according to different metabolic tumor phenotypes. Twenty-month and 60-month overall survival rates were calculated by the Kaplan-Meier method and log rank test for statistical significances. Chi-square test was used to compare
GLUT1 and mitochondrial superoxide dismutase (SOD2) up- or down-regulation in patient tumors. $P<0.05$ was considered statistically significant.

\section{Results}

\section{Institutional HNSCC Patient Cohort}

To study the interdependencies of tumor metabolism, TIME, and response to CRT, a retrospective HNSCC cohort consisting of 73 HNSCC patients (oral cavity, oropharynx, hypopharynx, larynx tumors) was established. Patient and tumor features are listed in Table 1, and hazard ratios (HR) for 5-year overall survival are listed in Table 2. Included patients were CRT-naive at the time of the biopsy and had received primary, nonsurgical treatment afterward consisting of cisplatin-based CRT at the Department of Radiotherapy, University Hospital Regensburg, from 2005 to 2010. Average Karnofsky performance score, available for 32 patients, was $80 \%$ (ranging from $50 \%$ to $100 \%$ ). Radiation was administered as intensity-modulated radiotherapy $(56 \%)$ or intensity-modulated arc therapy $(37 \%)$. The remainder received 3-dimensional radiotherapy (7\%). In $51 \%$ of cases, radiation was hyperfractionated/accelerated. Average total radiation dose was $69 \mathrm{~Gy}(\mathrm{SD}= \pm 3.3 \mathrm{~Gy}$ ). Additionally, all patients received $40 \mathrm{mg} / \mathrm{m}^{2}$ cisplatin with a mean cumulative dose of $181 \mathrm{mg}$ (SD $= \pm 60 \mathrm{mg}$ ). Four patients received additional doses of carboplatin, whereas for four patients, a dosage reduction of $50 \%$ to $75 \%$ had to be performed. Formalin-fixed, paraffin-embedded tumor tissues of the most recent biopsies before CRT initiation were used for histologic and immunohistochemical evaluations. All patients presented with an advanced stage of disease [90\% of patients with Union for International Cancer Control (UICC) stage IV, $10 \%$ of patients with UICC stage III]. Lymph node metastases were present in $83 \%$ of patients. The best clinical predictor of 5-year survival was $\mathrm{T}$ stage with a trend toward improved survival for patients with stage T2 [HR, 0.170; 95\% CI, 0.023-1.248; $P=0.081$ ]. Twelve patients (16.4\%) were treated for recurrences after prior surgical resection without CRT. P16 as a surrogate marker for HPV-related HNSCC was positive in seven cases (9.6\%) and showed a trend toward improved 5-year survival with a HR of 0.444 (95\% CI, 0.138-1.429; $P=0.173$ ). Patients with oral cavity carcinomas (HR, 1.575; 95\% CI, 0.743-3.338; $P=0.236)$ and moderately differentiated $\operatorname{HNSCC}(\mathrm{HR}, 1.492$; CI, $0.801-2.777 ; P=0.207$ ) showed a modest trend toward worse 5-year survival.

\section{Metabolic and Immunologic Characteristics of the HNSCC Patient Cohort}

All immunohistochemical analyses were performed on whole specimen sections of the most current biopsies before CRT initiation. To characterize the metabolic status, HNSCC were evaluated for COX5B and GLUT1 expression. GLUT1, which facilitates glucose transport across 
Table 1 Clinical and Pathological Characteristics of the HNSCC Cohort $(N=73)$

\begin{tabular}{|c|c|}
\hline Characteristic & Value \\
\hline Mean age, years & $59.9 \pm 10.5$ \\
\hline Sex, male & $67(91.8)$ \\
\hline p16 status, positive & $7(9.6)$ \\
\hline \multicolumn{2}{|l|}{ Anatomic site } \\
\hline Oropharynx & $25 \pm 34.2$ \\
\hline Oral cavity & $15 \pm 20.5$ \\
\hline Hypopharynx & $17 \pm 23.3$ \\
\hline Larynx & $16 \pm 21.9$ \\
\hline \multicolumn{2}{|l|}{ T stage $(n=72)$} \\
\hline 2 & $5 \pm 6.9$ \\
\hline 3 & $24 \pm 33.3$ \\
\hline 4 & $43 \pm 59.7$ \\
\hline \multicolumn{2}{|l|}{$\mathrm{N}$ stage $(n=72)$} \\
\hline 0 & $12 \pm 16.7$ \\
\hline 1 & $5 \pm 6.9$ \\
\hline 2 & $45 \pm 62.6$ \\
\hline 3 & $10 \pm 13.9$ \\
\hline Distant metastasis $(n=72)$ & $7 \pm 9.7$ \\
\hline \multicolumn{2}{|l|}{ UICC stage $(n=72)$} \\
\hline III & $7 \pm 9.7$ \\
\hline IV & $65 \pm 90.4$ \\
\hline \multicolumn{2}{|l|}{ Differentiation } \\
\hline Well & $2 \pm 2.7$ \\
\hline Moderate & $45 \pm 61.6$ \\
\hline Poor & $24 \pm 32.8$ \\
\hline \multicolumn{2}{|l|}{ Recurrence } \\
\hline After surgical treatment & $12 \pm 16.4$ \\
\hline Primary tumor & $61 \pm 83.6$ \\
\hline
\end{tabular}

Values are presented as means \pm SD and $n(\%)$.

HNSCC, head and neck squamous cell carcinoma; UICC, Union for International Cancer Control.

plasma membranes, serves as an indicator of anaerobic, glucose-dependent, and glycolytic tumor metabolism. ${ }^{18-20}$

COX5B, which is a subunit of the last enzyme in the mitochondrial electron transport chain, had shown differential expression patterns in a previous study of HPV-positive and -negative oropharyngeal squamous cell carcinomas, and was used as a marker for mitochondrial-rich, aerobic tumor metabolism. ${ }^{16}$

The mean expression intensity of all 73 cases according to the $\mathrm{H}$-score was 140 (SEM $= \pm 7.089)$ for COX5B and 160 (SEM $= \pm 4.573$ ) for GLUT1. HNSCC were further stratified into COX5B low and high tumors, as well as GLUT1 low and high tumors, according to the mean expression levels of all tumors.

To assess the TIME, HNSCC were evaluated for infiltration by $\mathrm{CD} 8^{+} \mathrm{CTL}$ and $\mathrm{CD} 4^{+} \mathrm{T}$ helper cells. Average number of $\mathrm{CD}^{+} \mathrm{CTL}$ and $\mathrm{CD} 4^{+} \mathrm{T}$ helper cells per tumor high power field was $19(\mathrm{SEM}= \pm 3.638)$ and $16(\mathrm{SEM}= \pm 1.861)$, respectively. As an indicator of antitumor immune response, the CD8/ CD4 ratio was calculated, with a mean ratio of 1.4 (SEM $= \pm 0.199$ ). HNSCC were further stratified into CD8/ $\mathrm{CD} 4$ low and high tumors according to the mean $\mathrm{CD} 8 / \mathrm{CD} 4$ ratio of all tumors.
Correlating metabolic with immunologic characteristics, HNSCC with high COX5B expression presented with a trend toward an increased CD8/CD4 ratio $(P=0.0745)$. Vice versa, tumors with high GLUT1 expression had a significantly decreased $\mathrm{CD} 8 / \mathrm{CD} 4$ ratio $(P=0.0206)$ (Figure $1, \mathrm{~A}, \mathrm{~B}, \mathrm{E}$, and F). Additionally, COX5B high tumors also had a significantly increased number of tumor-infiltrating forkhead box $\mathrm{P}^{+}$regulatory $\mathrm{T}$ cells (Tregs). GLUT1 expression, by contrast, had no impact on infiltration by Tregs (Figure 1, C, $\mathrm{D}, \mathrm{G}$, and $\mathrm{H}$ ).

\section{Predictive and Prognostic Features of Metabolic and Immune Markers and Marker Combinations}

Twenty-month overall survival (short-term survival is considered an indicator of initial therapy response) and 60-month overall survival were analyzed by Cox regression (Table 3 ) and Kaplan-Meier method (Figure 2). Mean survival time was 30.1 months (95\% CI, 25.0-35.1). In separate evaluations of immune and metabolic markers, the $\mathrm{CD} 8 / \mathrm{CD} 4$ ratio provided the best information on short-term survival, with a trend toward an increased HR (HR, 1.825, 95\% CI, 0.793-4.197; $P=0.157$ ) for patients with a decreased CD8/CD4 ratio. Interestingly, patients with low tumor GLUT1 expression presented with

Table 2 Hazard Ratios (HR) of 60-Month Survival for Clinical and Pathological Characteristics of the HNSCC Cohort

\begin{tabular}{|c|c|c|}
\hline Characteristic & $\mathrm{HR}(\mathrm{CI})$ & $P$ value \\
\hline Age, $\leq 60$ vs. $>60$ years & $0.78(0.445-1.369)$ & 0.387 \\
\hline Sex, male vs. female & $0.665(0.264-1.675)$ & 0.386 \\
\hline $\begin{array}{l}\text { p16 status, positive vs. } \\
\text { negative }\end{array}$ & $0.444(0.138-1.429)$ & 0.173 \\
\hline \multicolumn{3}{|l|}{ Anatomic site } \\
\hline Oropharynx & 1 & 0.54 \\
\hline Oral cavity & $1.575(0.743-3.338)$ & 0.236 \\
\hline Hypopharynx & $1.034(0.498-2.147)$ & 0.93 \\
\hline Larynx & $0.869(0.401-1.884)$ & 0.722 \\
\hline \multicolumn{3}{|l|}{ T stage $(n=72)$} \\
\hline 2 & $0.170(0.023-1.248)$ & 0.081 \\
\hline 3 & $0.954(0.524-1.734)$ & 0.876 \\
\hline 4 & 1 & 0.219 \\
\hline \multicolumn{3}{|l|}{$\mathrm{N}$ stage $(n=72)$} \\
\hline 0 & $0.931(0.510-1.701)$ & 0.816 \\
\hline 1 & $0.891(0.366-2.168)$ & 0.799 \\
\hline 2 & $1.074(0.678-1.700)$ & 0.762 \\
\hline 3 & 1 & 0.969 \\
\hline Distant metastasis $(\mathrm{n}=72)$ & $1.633(0.692-3.852)$ & 0.263 \\
\hline \multicolumn{3}{|l|}{ UICC stage $(n=72)$} \\
\hline III & $0.898(0.356-2.265)$ & 0.82 \\
\hline IV & 1 & \\
\hline \multicolumn{3}{|l|}{ Differentiation } \\
\hline Well & $0.573(0.075-4.364)$ & 0.591 \\
\hline Moderate & $1.492(0.801-2.777)$ & 0.207 \\
\hline Poor & 1 & 0.44 \\
\hline Recurrence vs. primary tumor & $0.669(0.314-1.425)$ & 0.298 \\
\hline
\end{tabular}

HNSCC, head and neck squamous cell carcinoma; UICC, Union for International Cancer Control. 

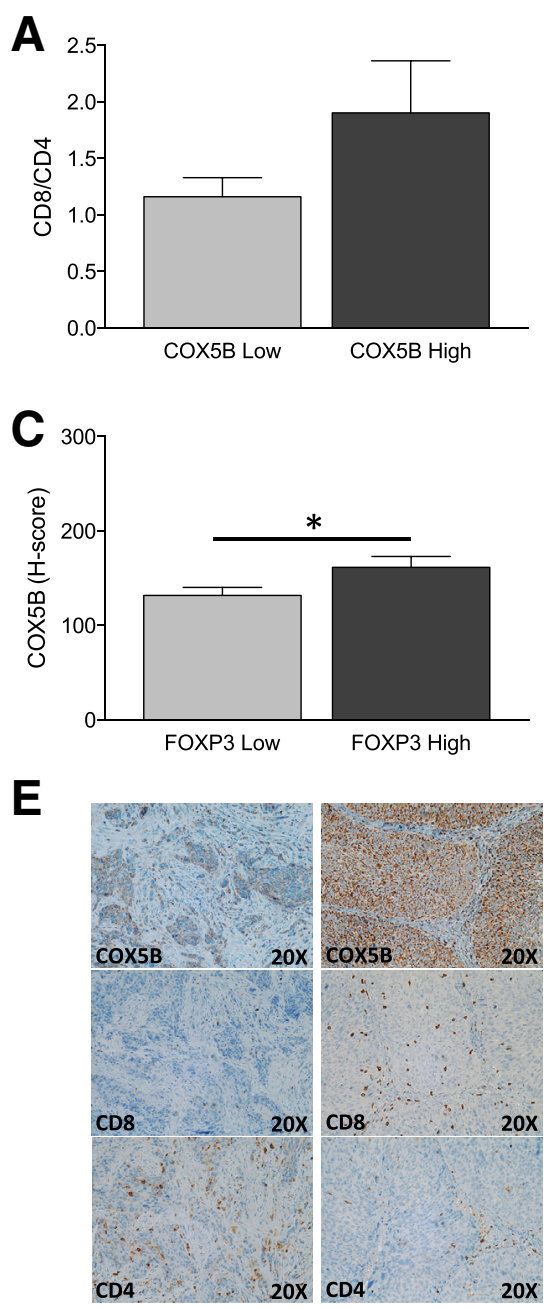

G

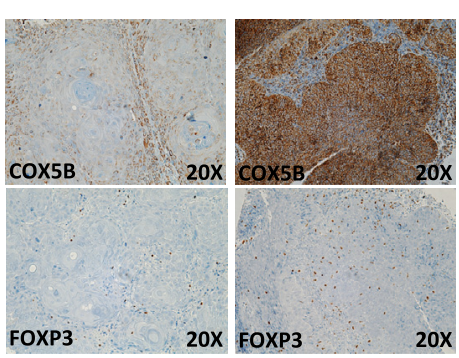

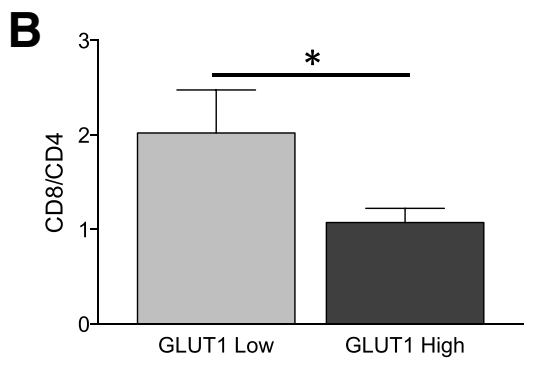
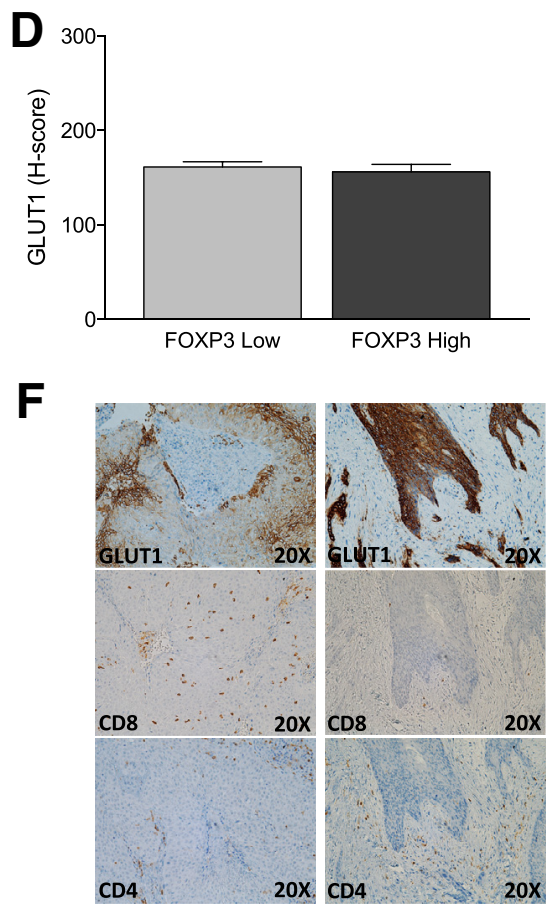

H

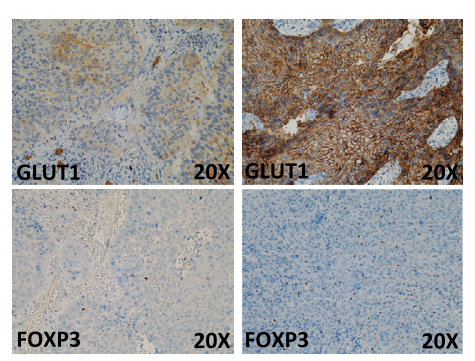

Figure 1 Distinct and opposing tumor immune profiles in mitochondrial-rich and glucosedependent head and neck squamous cell carcinoma. A: Chart diagram of average CD8/CD4 ratio in cytochrome $c$ oxidase subunit 5B (COX5B) low and COX5B high tumors trending toward higher antitumor immune response (CD8/CD4) in mitochondrial-rich ( $\mathrm{CO} 5 \mathrm{~B}$ high) tumors. B: Chart diagram of average $\mathrm{CD} 8 / \mathrm{CD} 4$ ratio in glucose transporter 1 (GLUT1) low and GLUT1 high tumors with significantly decreased antitumor immune response (CD8/CD4) in glucose-dependent (GLUT1 high) tumors. C: Chart diagram of significantly increased average COX5B expression in tumors with high infiltration by forkhead box P3 (FOXP3) ${ }^{+}$ lymphocytes. D: No difference in GLUT1 expression in tumors with weak and strong infiltration by FOXP3 ${ }^{+}$lymphocytes. E: Immunohistochemical images of two representative tumors with low COX5B, low CD8 and high CD4 or high COX5B, high CD8 and low CD4. F: Immunohistochemical images of two representative tumors with low GLUT1, high CD8 and low CD4 or high GLUT1, low CD8 and high CD4. G: Immunohistochemical images of two representative tumors with low $\mathrm{COX} 5 \mathrm{~B}$ and low FOXP3 or high COX5B and high FOXP3. H: Immunohistochemical images of two representative tumors with low GLUT1 and low FOXP3 or high GLUT1 and intermediate FOXP3. Data are expressed as means \pm SEM (A-D). ${ }^{*} P<0.05$. significantly worse long-term survival (HR, 1.753, 95\% CI, 1.010-3.043; $P=0.046$ ).

Because we hypothesized that an active antitumor immune response can only emerge in the presence of a favorable metabolic TME, we analyzed the effect of different immune and metabolic marker combinations on short- and long-term survival. We anticipated that a combination of an aerobic, mitochondrial-rich (high COX5B) or glucose-independent (low GLUT1) tumor metabolism together with a CD8dominant tumor immune infiltrate (high CD8/CD4 ratio) would represent TMEs predisposing to a strong therapyrelated antitumor immune response leading to improved CRT efficacy. Only eight and nine of 73 patients, respectively, had an increased $\mathrm{CD} 8 / \mathrm{CD} 4$ ratio together with high COX5B or low GLUT1 expression (Figure 2, A and B). Short-term survival was better in tumors combining high CD8/CD4 together with high COX5B $(P=0.053)$ or low GLUT1 $(P=0.033$ ) as compared to all other tumors (Figure 2, $\mathrm{C}$ and D). Combining the two metabolic markers with CD8/CD4 ratio, a subset of 14 patients may be defined that is characterized by a favorable immune and metabolic TME (high CD8/CD4 and high COX5B or low GLUT1), who demonstrated an excellent outcome with a short-term survival fraction of $85.7 \%$ as compared to all other patients with a survival fraction of $45.8 \%(P=0.008)$ (Figure 2, E and F). With regard to long-term survival, a significantly improved 
Table 3 Hazard Ratios (HR) of 20-Month and 60-Month Survival for Immune and Metabolic Markers and Marker Combinations

\begin{tabular}{lccc}
\hline Markers and marker combinations & 20-month HR (CI) & $P$ value & 60 -month HR (CI) \\
\hline Single markers & & & \\
Immunological markers & $1.389(0.648-2.978)$ & 0.398 & $1.177(0.644-2.151)$ \\
$\quad$ CD8 (low vs. high) & $0.809(0.400-1.636)$ & 0.555 & $0.806(0.454-1.432)$ \\
CD4 (low vs. high) & $1.825(0.793-4.197)$ & 0.157 & $1.068(0.584-1.954)$ \\
CD8/CD4 (low vs. high) & $0.827(0.411-1.663)$ & 0.594 & $0.872(0.492-1.544)$ \\
F0XP3 (low vs. high) & & & 0.597 \\
Metabolic markers & $1.265(0.617-2.597)$ & 0.521 & $0.729(0.418-1.271)$ \\
COX5B (low vs. high) & $1.276(0.644-2.526)$ & 0.485 & $1.753(1.010-3.043)$ \\
GLUT1 (low vs. high) & & & 0.638 \\
Marker combinations & $5.563(0.739-41.866)$ & 0.096 & 0.265 \\
COX5B vs. CD8/CD4 & $5.026(0.605-41.770)$ & 0.135 & 0.046 \\
COX5B low CD8/CD4 low & $6.347(0.811-49.650)$ & 0.078 & $1.241(0.441-3.490)$ \\
COX5B low CD8/CD4 high & 1 & 0.37 & $1.715(0.670-4.390)$ \\
COX5B high CD8/CD4 low & 1 & 1 \\
COX5B high CD8/CD4 high & $11.097(1.448-85.063)$ & 0.021 & 0.958 \\
GLUT1 vs. CD8/CD4 & 1 & 0.683 \\
GLUT1 low CD8/CD4 low & $4.608(0.605-35.064)$ & 0.14 & 0.261 \\
GLUT1 low CD8/CD4 high & $5.996(0.722-49.814)$ & 0.097 & 0.382 \\
GLUT1 high CD8/CD4 low & & $0.273(0.939-5.506)$ \\
GLUT1 high CD8/CD4 high & & 1 \\
\hline
\end{tabular}

COX5B, cytochrome c oxidase subunit 5B; FOXP3, forkhead box P3; GLUT1, glucose transporter 1.

outcome was demonstrated for the subset of patients with a high CD8/CD4 ratio and low GLUT1 within the low GLUT1 patient group $(P=0.026)$ (Table 3$)$. There was, however, no significant difference in long-term survival for the subgroup of patients with high CD8/CD4 and low GLUT1 or high COX5B in comparison to all other patients.

\section{TCGA Database Analysis of Immune and Metabolic Gene Signatures Correlating with HNSCC Survival after CRT}

Next, the prognostic value of a favorable immune and metabolic TME was evaluated in a HNSCC cohort of the TCGA database. At the time of the most recent update (August 2016), the provisional TCGA HNSCC data set included survival and gene expression information on 522 patients (Supplemental Table S1). Expression of genes coding for proteins with predictive value in the initial cohort (CD8A, COX5B, GLUT1) were queried across the TCGA HNSCC cohort. According to mean expression levels of the respective genes $C D 8 A, G L U T 1$, and $C O X 5 B$ (mRNA zscore $=0$ ) patients were stratified into high and low geneexpressing groups. $C D 8 A$ was up-regulated in 144 patients (27.59\%). Although $C O X 5 B$ expression was equally balanced in $C D 8 A$ high and low groups, GLUT1 was significantly more frequently up-regulated in $C D 8 A$ low than $C D 8 A$ high patients $(50.0 \%$ vs. $27.8 \% ; P<0.0001)$ (Figure 3A). Mean overall survival time for all patients was 40.1 months (95\% CI, 37.9-42.2). Evaluating genes separately, only increased CD8A expression significantly contributed to improved long-term survival $(P=0.049)$ (Figure 3, B-D). Next, patients were stratified according to favorable versus unfavorable immune and metabolic gene signatures, with a favorable signature defined by high $C D 8 A$, high $C O X 5 B$, and low GLUT1, and an unfavorable signature defined by low $C D 8 A$, low $C O X 5 B$, and high GLUT1. A favorable gene signature was found in 41 patients $(7.87 \%)$, and an unfavorable gene signature in 132 patients $(25.34 \%)$. Patients with a favorable immune and metabolic gene signature presented with markedly improved short-term $(P=0.013)$ and long-term $(P=0.048)$ survival (Figure $3 \mathrm{E})$.

Because we hypothesized that part of the beneficial outcome of tumors with high $C D 8 A$, high $C O X 5 B$, and low GLUT1 can be attributed to the proapoptotic and immunemodulatory effects of ROS, the levels of SOD2 were next assessed in tumors with favorable and unfavorable immune and metabolic gene signatures. This mitochondrial enzyme catalyzes the conversion of superoxide $\left(\mathrm{O}_{2}^{-}\right)$into hydrogen peroxide, which is an important intracellular messenger and involved, among others, in induction of apoptosis and lymphocyte activation. ${ }^{21,22}$ The percentage of tumors with SOD2 up-regulation was significantly higher in the favorable immune and metabolic group $(46.3 \%)$ as compared to the unfavorable group (29.5\%; $P=0.0466$ ) (Figure $3 \mathrm{~F}$ ).

\section{Glycolysis Inhibition and Radiation Synergistically Induce Reactive 0xygen Production In Vitro and Induce Chemotaxis of Immune Cells toward UM-SCC-47 Spheroids}

An effective CRT response relies on radiation-induced ROS production. $^{23,24}$ ROS, particularly hydrogen peroxide, are important messengers of tumor cell death and antitumor immune response. ${ }^{25}$ Mitochondria are the main source of ROS production. Therefore, the degree of radiation-induced ROS accumulation was hypothesized to depend on the metabolic phenotype 

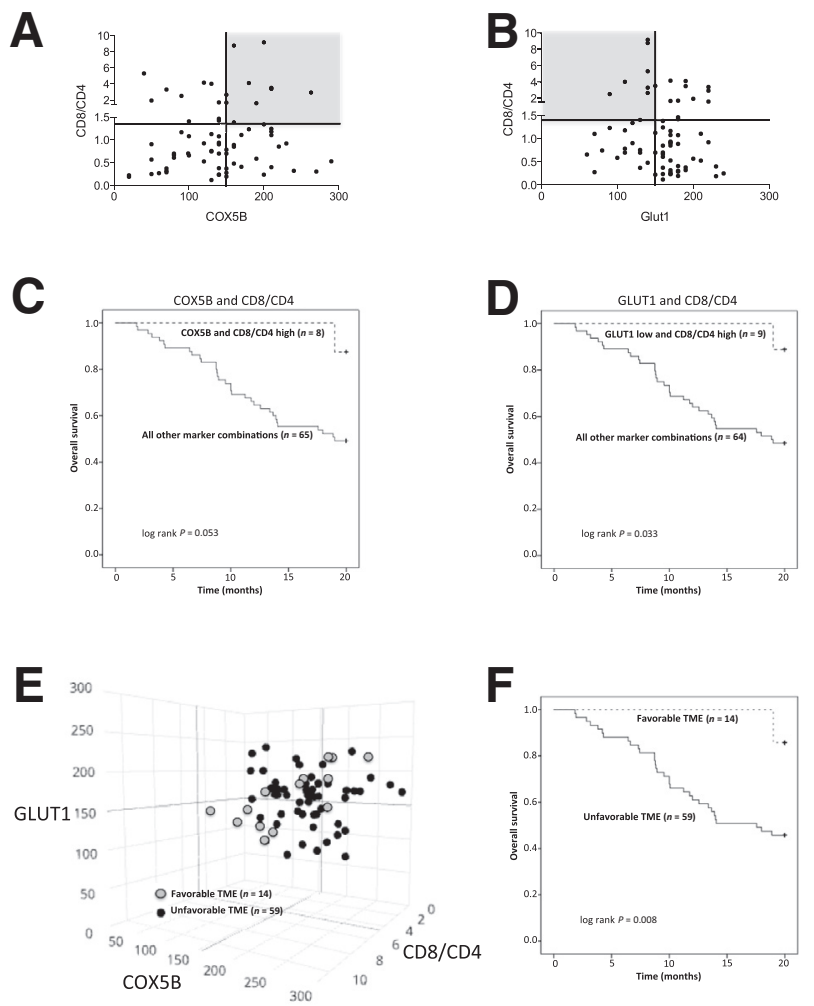

Figure 2 Metabolic and immune marker combinations predict response to chemoradiotherapy (CRT) in head and neck squamous cell carcinoma (HNSCC). A and B: Four quadrant stratification of tumors according to mean $\mathrm{CD} 8 / \mathrm{CD} 4$ ratio (horizontal line) and mean cytochrome $\mathrm{c}$ oxidase subunit $5 \mathrm{~B}$ (COX5B) or glucose transporter 1 (GLUT1) expression (vertical lines). $\mathrm{C}$ and D: HNSCC with high CD8/CD4 and high COX5B or high CD8/CD4 and low GLUT1 (grayed-out quadrants) have a significantly improved response to CRT as represented by 20 -month survival. E: Three-dimensional scatter blot of HNSCC according to CD8/CD4 ratio, COX5B expression, and GLUT1 expression. Tumors with high CD8/CD4 ratio and high COX5B or low GLUT1 expression [favorable immune and metabolic tumor microenvironment (TME) types] are marked in gray. F: Favorable immune and metabolic TME types present with a drastically improved response to CRT.

of a tumor. 2-Deoxyglucose (DGL) is a glucose analog that inhibits glycolysis via competitive binding to hexokinase, the rate-limiting step of glucose metabolism. UM-SCC-47 tumor cells were grown in the presence or absence of $10 \mu \mathrm{mol} / \mathrm{L}$ DGL for 4 days. DGL-treated and untreated tumor cells were labeled with $2^{\prime}, 7^{\prime}$-dichlorofluorescin diacetate (DCFDA) to visualize ROS production and exposed to 10-Gy X-ray radiation. X-ray radiation $(10 \mathrm{~Gy})$ led to a survival fraction of $50 \%$ of UM-SCC47 according to clonogenic assay (Supplemental Figure S2). ROS production was significantly higher in cells cultured in the presence of DGL or in radiated cells $(P<0.0005)$. The exposure of DGL-treated cells to radiation further increased ROS production $(P=0.0463)$ (Figure 4, A and B).

On the basis of these findings, the effect of DGL treatment and radiation on chemotaxis of immune cells toward 3-dimensional UM-SCC-47 tumor spheroids was investigated. UM-SCC-47 were grown for 4 days in the presence or absence of $10 \mu \mathrm{mol} / \mathrm{L}$ DGL and subsequently exposed to 10-Gy X-ray radiation. Afterward, PBMCs isolated from healthy human donors were fluorescently labeled and added to treated tumor spheroids. Because it was hypothesized that ROS secretion by treated spheroids might play a role in mediating chemotaxis, the optimal time point was determined in preliminary experiments by assessing PBMC migration at 1 hour, 3 hours, 5 hours, 12 hours, and 24 hours. In subsequent experiments, migration of PBMCs toward spheroids was measured after 5 hours with a fluorescence microscope. Although DGL treatment had no effect on PBMC attraction, and 10-Gy radiation slightly increased the number of PBMCs migrating toward tumor spheroids, spheroid growth in the presence of DGL followed by radiation exposure caused a significantly higher chemotaxis of PBMCs to spheroids (Figure 4, C and D). To elucidate the role of DGL and radiation-induced ROS production in PBMC chemotaxis, ROS production was inhibited via the ROS scavenger $N$-acetylcysteine (NAC). ${ }^{26}$ Addition of $10 \mathrm{mmol} / \mathrm{L}$ NAC before PBMC spheroid co-cultures completely abrogated the effect of immune cell chemotaxis to below baseline (Figure 4D).

\section{Discussion}

In this study, the interdependencies of the TIME and tumor metabolism in HNSCC and how these characteristics affect survival in HNSCC patients was assessed. Over the last decades, the tumor immune profile has gained more and more importance as a predictive and prognostic factor in solid tumors, including the recognition of CRT as an inducer of an antitumor immune response. The pre-existing TIME of HNSCC is highly variable and rendered dysfunctional by tumor-associated immunosuppression and immune escape. ${ }^{27}$ In this scenario, an unfavorable equilibrium of protumor components, such as myeloid-derived suppressor cells, Tregs, tumor-associated macrophages or eosinophils, and antitumor components such as $\mathrm{CD}^{+} \mathrm{CTL}$, $\mathrm{CD}^{+} \mathrm{T}$ helper cells, which can have protumor and antitumor activities, natural killer cells, and dendritic cells, preclude successful tumor elimination. Correspondingly, the character of the local tumor immune response strongly impacts therapy efficacy and outcome. ${ }^{3,28}$ In particular, a preexisting $\mathrm{CD}^{+}{ }^{+} \mathrm{CTL}$ dominant tumor immune infiltrate has been shown in many different solid tumors to be beneficial for survival and therapy response. ${ }^{29-32}$ Due to the high variability of different tumor infiltrating immune cell subtypes, ratios between T-cell subsets have been shown to provide even better information on patient survival. ${ }^{33}$ In this study, the tumor infiltrating CD8/CD4 ratio was used to define tumors with a CD8-dominant antitumor immune infiltrate.

To characterize the tumor metabolism, the expression of two proteins involved in energy metabolism was assessed: GLUT1 and COX5B. GLUT1 is widely accepted as a surrogate marker of glycolytic or glucose-dependent metabolism. Increased GLUT1 expression in HNSCC and esophageal cancers has been associated with more 

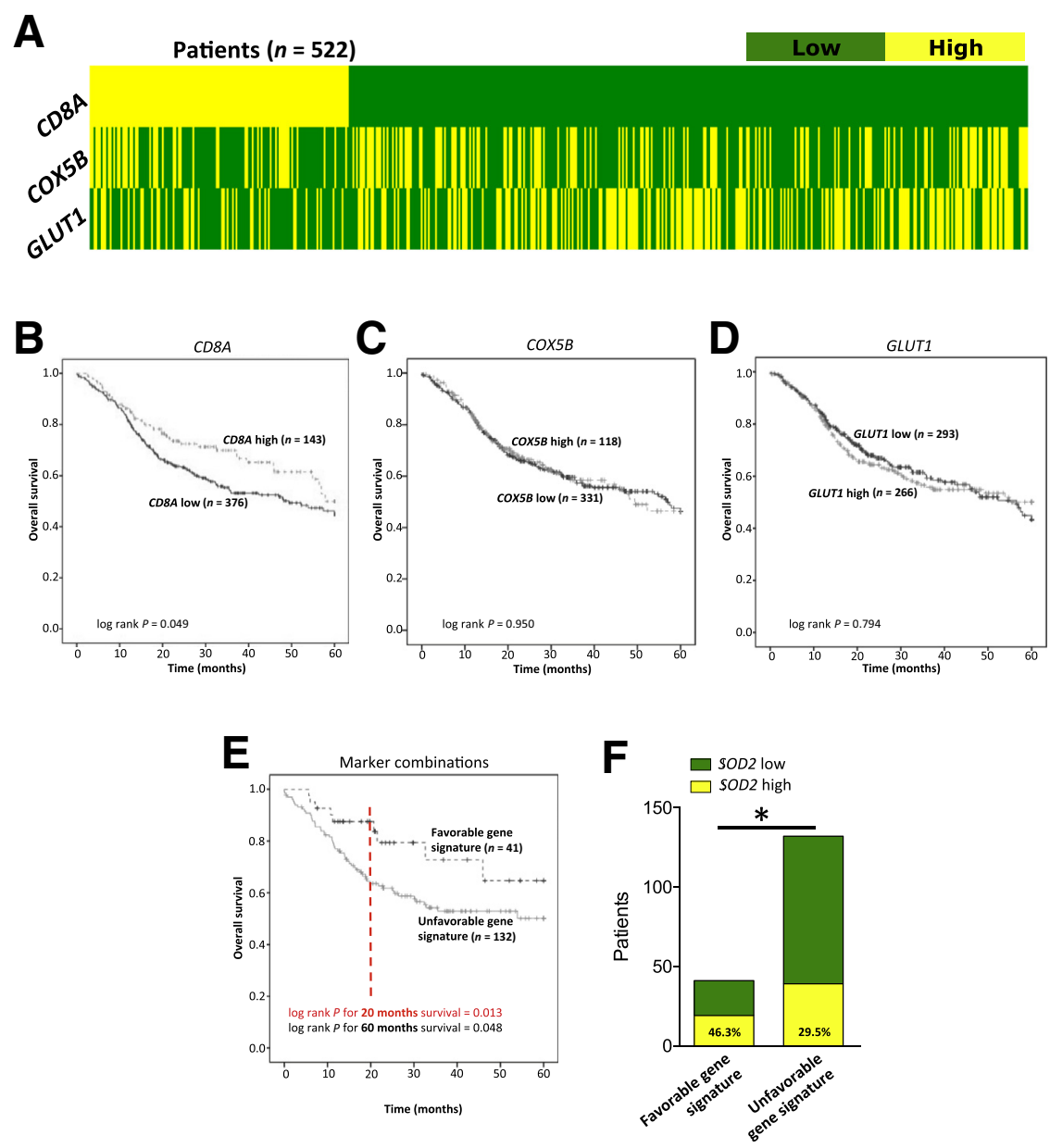

Figure 3 CD8 cytotoxic T cells-dominant tumor immune microenvironment inversely correlates with glucose-dependent tumor metabolism and allows selection of patients with a favorable immune and metabolic gene signature in The Cancer Genome Atlas head and neck squamous cell carcinoma (HNSCC) data cohort. A: Heat map of down-regulated (green) and up-regulated (yellow) gene expression levels of CD8A, cytochrome $c$ oxidase subunit $5 B$ (COX5B), and glucose transporter 1 (GLUT1) according to mean expression levels (mRNA z-score $=0)$. Patients with high CD8A have significantly more often down-regulated GLUT1 expression levels $(72.1 \%$ vs. $50.0 \%, P<0.0001)$. There is no association between CD8A and COX5B expression levels. B-D: Kaplan-Meier curves showing improved long-term overall survival only for HNSCC patients with increased CD8A expression levels (B), whereas COX5B and GLUT1 expression levels individually have no effect on overall survival (C and D). E: A subgroup of patients with a favorable immune and metabolic gene signature (high CD8A, high COX5B, low GLUT1) shows significantly improved short-term survival/therapy response (20 months) and long-term survival (60 months) as compared to patients with an unfavorable immune and metabolic gene signature (low CD8A, low COX5B, high GLUT1). F: The expression level of the hydrogen peroxide-producing enzyme superoxide dismutase 2 (SOD2) is significantly more often up-regulated in tumors with a favorable gene signature than tumors with an unfavorable gene signature and imply a role for reactive oxygen species-mediated signaling. ${ }^{*} P<0.05$. aggressive growth and poorer survival in several studies. $^{20,34}$ As a marker for a mitochondrial-rich and aerobic tumor metabolism, COX5B, which is a subunit of the cytochrome c oxidase complex, also known as Complex IV, was used. This is the last enzyme in the mitochondrial electron transport chain. We and others have already shown increased expression of COX5B in HNSCC and other epithelial malignancies. ${ }^{16,35,36}$ A glucose-dependent tumor metabolism as defined by high GLUT1 expression is typically associated with an accumulation of lactate as end product of aerobic glycolysis due to the Warburg effect. ${ }^{37}$ In a mitochondrial-rich tumor metabolism, on the other hand, a decrease in lactate is expected, as pyruvate or lactate is shuttled into the Krebs cycle and respiratory chain. The amount of lactate in the TME of these different metabolic phenotypes is crucial, because it affects patient outcome. For example, solid tumors with high intratumoral lactate levels have been shown to metastasize more frequently and to be associated with a decreased overall survival as compared to tumors with low lactate levels. ${ }^{38}$ Besides stimulating several proangiogenic and migratory factors as transforming growth factor- $\beta 2$ and vascular endothelial growth factor, lactate also impacts tumor immune escape and response to CRT. Lactate inhibits cytokine release from dendritic cells and inhibits $\mathrm{CD}^{+}$CTLs. Activated $\mathrm{CD}^{+}$ CTLs rely on glycolysis for energy production and secretion of lactate into the extracellular space to maintain a physiological $\mathrm{pH}$. In a lactate-rich TME, they are therefore not capable of secreting accumulated lactate, which results in a decrease of intracellular $\mathrm{pH}$ and consecutive dysfunction of their cytotoxic activity. ${ }^{13}$ Additionally, lactate levels have been positively correlated with radioresistance in HNSCC. ${ }^{15}$ This may be due to the antioxidant capacities of lactate as a ROS scavenger. ROS, on the other hand, are crucial for effective chemotherapy or radiation-induced tumor cell DNA damages because they bind and fixate DNA strand breaks. $^{39}$

In the correlative analyses of tumor-infiltrating lymphocytes and metabolic phenotypes in HNSCC, a significant interdependence of tumor metabolism with the TIME was identified. Corresponding to the inhibitory effect of a glycolytic tumor metabolism on the cytotoxic tumor immune response, the CD8/CD4 ratio was significantly lower in tumors with high GLUT1 expression as opposed to tumors with low GLUT1 expression. Mitochondrial-rich tumors with high COX5B expression, on the other hand, were also associated with higher $\mathrm{CD} 8 / \mathrm{CD} 4$ ratios, suggesting that an aerobic tumor metabolism provides a TIME, which is more 

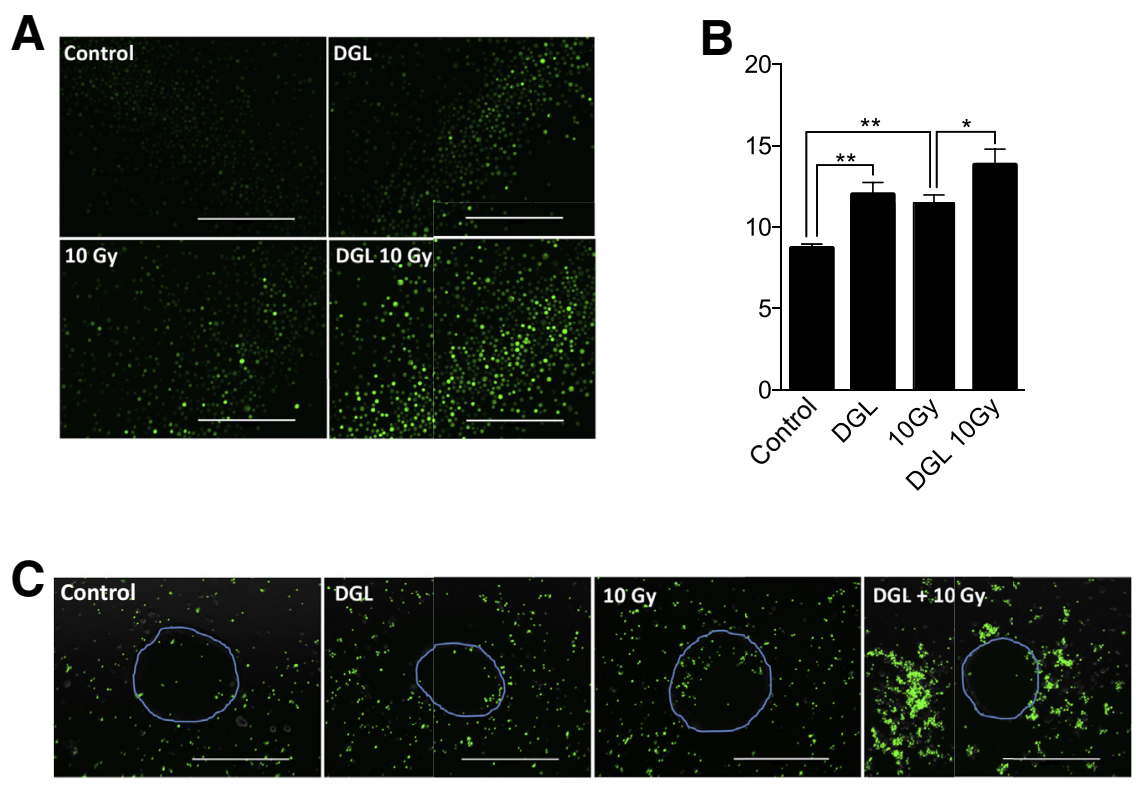

\section{+ NAC}

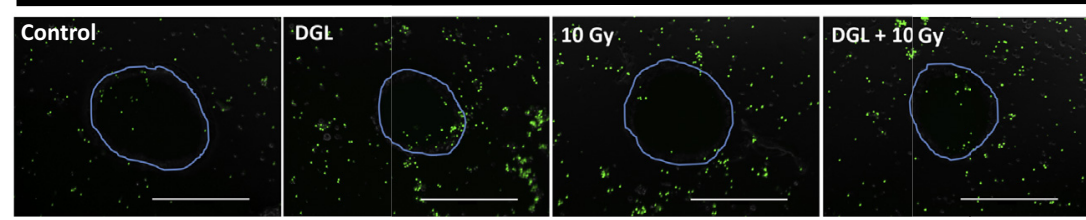

D

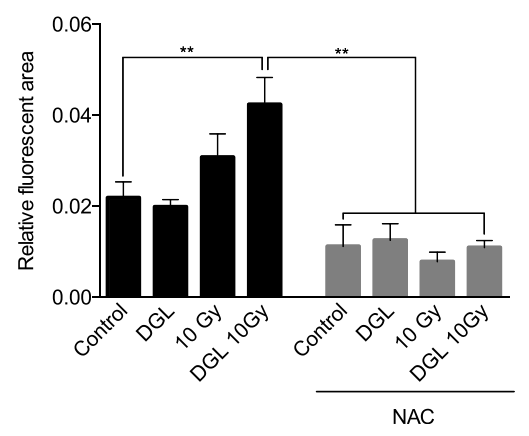

permissive for a cytotoxic antitumor immune response. Additionally, COX5B was significantly higher in tumors with a strong Treg infiltrate as compared to tumors with only a few Tregs. The metabolism of Tregs is unique in that they use oxygen-dependent fatty acid oxidation for energy production. ${ }^{40}$ Therefore, it is assumed that only tumors with an aerobic metabolism provide the necessary oxygen supply to allow Treg infiltration. Regarding the high CD8/CD4 ratio in nonglycolytic and aerobic tumors, we have already observed very similar features of a dominant mitochondrial-rich and CD8/CD4 high TME in HPV-positive OPSCC as compared to HPV-negative ones. ${ }^{16}$ Because HPV-positive OPSCC are known for their excellent prognosis and response to CRT, there is a strong suspicion that TMEs featuring these characteristics generally present with an improved response to therapy and better survival.
Figure 4 Radiation and inhibition of glycolysis cause reactive oxygen species (ROS)-dependent peripheral blood mononuclear cells (PBMCs) chemotaxis toward head and neck squamous cell carcinoma (HNSCC) spheroids. A and B: 2-Deoxyglucose (DGL) treatment and radiation induce an increase of ROS production in UM-SCC-47 cells. Tumor cells were grown for 4 days in the presence or absence of DGL. After H2DCFDA (ROS probe) labeling, cells were exposed to 10-Gy radiation or mock treatment, and ROS production was measured under a fluorescence microscope ( $\mathbf{A}$, representative images) followed by ImageJ software version $1.49 \mathrm{v}$ (NIH, Bethesda, MD; http:// imagej.nih.gov/ij) quantification of fluorescence intensity (B). C and D: DGL treatment combined with radiation exposure leads to increased PBMC chemotaxis toward UM-SCC-47 spheroids (spheroid borders are depicted in blue). Tumor cells were grown in the presence or absence of DGL for 4 days and transferred into agarose-coated wells. After 10-Gy radiation or mock treatment, spheroids were co-cultured with carboxyfluorescein succinimidyl ester (CFSE)-labeled human PBMC, and PBMC migration was imaged under a fluorescence microscope 5 hours after coculture initiation. DGL-treated spheroids attracted significantly more PBMCs after radiation exposure than control spheroids (D). Addition of the ROS scavenger $\mathrm{N}$-acetylcysteine (NAC) abrogated this effect, indicating a ROS-related mechanism. All spheroid/PBMC co-culture experiments were performed in triplicates Data are expressed as means $\pm \mathrm{SEM}$ (B and D). $n=3$ (B, experiments). ${ }^{*} P<0$. $05 ;{ }^{*} P<0.01$. Scale bar $=400 \mu \mathrm{m}$. Original magnification, $\times 10$.
The tumors of our (predominantly HPV-negative) cohort were therefore stratified according to their metabolic and immunologic TME features and short-term survival (20 months) was assessed, which provides information on CRT response, as well as long-term survival (60 months). Only $11 \%$ and $12 \%$ of all patients, respectively, showed an immune and metabolic tumor phenotype characterized by a high $\mathrm{CD} 8 / \mathrm{CD} 4$ ratio together with a mitochondrial-rich or glucose-independent tumor metabolism. The respective tumors presented with a significantly improved short-term survival. Because the cases of patients with a favorable immune and metabolic tumor phenotype were fairly small in our own cohort, statistical bias due to disproportionate numbers cannot be excluded.

Therefore, mRNA data from the HNSCC collective of the TCGA data bank was used to assess the significance of a 
favorable immune and metabolic tumor phenotype in a bigger, independent cohort. In contrast to our own cohort, this collective includes 522 patients of all stages treated with primary surgery with or without CRT. In analogy to the results of our cohort, CD8A expression inversely correlated with GLUT1 expression. Although neither GLUT1 nor COX5B mRNA levels alone can predict survival, patients with increased $C D 8 A$ presented with a better prognosis. The best information on long-term and short-term survival may be achieved by combining immune and metabolic mRNA expression levels. An improved outcome for patients with a favorable immune and metabolic tumor phenotype (high CD8A, high COX5B, low GLUT1) as compared to an unfavorable phenotype (low $C D 8 A$, low $C O X 5 B$, high GLUTI) was confirmed.

In perspective, we think that our marker combination may constitute a helpful tool to distinguish HNSCC patients who would benefit from a CRT-based treatment approach, instead of a surgical therapy, in particular because there still exist no reliable clinical, histological or immunohistochemical features that can select patients with particularly CRT-sensitive tumors.

Oxidative stress is an important signal transduction mechanism of radiation- and chemo-induced apoptosis, and end effector of radiation damage with significant implications for response to radiotherapy and CRT. ${ }^{24,41}$ Therefore, we hypothesized that ROS, such as super oxide $\left(\mathrm{O}_{2}^{-}\right)$or hydrogen peroxide may also constitute important mediators between tumor metabolism and TIME contributing to the good prognosis of tumors with a favorable immune and metabolic TME. A comparison of SOD2 mRNA expression in the TCGA HNSCC cohort subgroups of favorable and unfavorable immune and metabolic patients supports a strong association between up-regulated SOD2 and favorable immune and metabolic tumors; and down-regulated SOD2 and unfavorable immune and metabolic tumors. SOD2 catalyzes the conversion of $\mathrm{O}_{2}^{-}$to hydrogen peroxide. Hydrogen peroxide is not only necessary for the fixation of DNA strand breaks, but is also involved in the initiation of redox-sensitive signaling cascades such as the MAPK/ERKpathway. ${ }^{42,43}$ High SOD2 levels have been shown to increase radiosensitivity in vivo. ${ }^{44}$

To investigate the connection of tumor metabolism and immune cells in the context of radiation-induced ROS production, a 3-dimensional spheroid model of UM-SCC-47 cells, which was metabolically modulated via the glucose inhibitor DGL, was used. First, the changes in ROS production via metabolic modulation and radiation were examined. As previously shown, the extended inhibition of glycolysis by DGL for 96 hours or exposure to radiation both caused an increase in ROS production. ${ }^{45}$ Additionally, radiation of DGL-treated tumor cells increased ROS production even further. DGL has already been shown to radiosensitize tumor cells in vitro and reduce tumor growth in vivo together with radiation. ${ }^{46}$ Several clinical trials of malignant glioma patients also showed an increase in survival for patients treated with DGL and radiation. ${ }^{47}$ The effect of DGL- and radiation-treated UM-SCC-47 tumors was consecutively assessed on the chemotaxis of human immune cells. In a tumor spheroid/PBMC co-culture model, we showed an increased migration of immune cells toward DGL-treated spheroids after radiation exposure. The complete abrogation of the immune cell migration toward the spheroid by the ROS scavenger NAC suggests this effect to be ROS and oxidative stress related. Supporting our observations, it has already been shown that chemokine-induced T-cell trafficking depends on the uptake of hydrogen peroxide by $\mathrm{T}$ cells. ${ }^{48}$ Another group demonstrated that treatment with an SOD2 mimic leads to radiosensitization and increased macrophage influx in a murine xenograft HNSCC tumor model. ${ }^{49}$

The interaction of tumor metabolism and tumor immune infiltrate is, however, complex and dependent on many different factors. For example, immune checkpoint modulators represent a potential mechanism of metabolism-mediated antitumor immune response. To assess the effect of changes in GLUT1 expression on immune checkpoint molecules, a biological network analysis was performed, which revealed network-wide changes of most key immune checkpoint molecules in response to increased GLUT1 levels. Evaluation of the network identified GLUT1-mediated control of AKT as a mediator of nuclear factor $\kappa \mathrm{B}$ signaling resulting in changes in immune checkpoint molecule expression (Supplemental Figure S3). Interestingly, it was just recently shown that aerobic glycolysis and lactate are features of acquired radioresistant cells and that this is an AKTmediated phenomenon, because radioresistance was reversed by AKT inhibitors. ${ }^{19}$ Our initial network studies point toward new targets to further investigate the interactions of tumor metabolism and antitumor immune response.

One weakness of the study is the patient selection of the HNSCC cohort treated by primary CRT. The patients included are generally characterized by progressed tumors and overall bad health status including operability, disease stage, comorbidities, etc. This is reflected in our cohort by a majority of UICC stage IV patients $(90.4 \%)$ and the remainder of patients with an UICC stage III, as well as an average Karnofsky performance status of $80 \%$. Furthermore, $12 \%$ patients were CRT-naive, but suffered from recurrences, which might behave differently under CRT than primary tumors. In Germany, primary CRT is typically reserved for advanced cases with palliative approaches, as well as for tumors that cannot be resected due to their anatomic location, or for patients whose comorbidities do not allow surgery. Therefore, this cohort is biased toward patients with rather poor prognoses as demonstrated by a mean overall survival of 30.1 months. Hence, multiple factors beyond tumor biology contribute to outcome and probably have a bigger effect on longterm survival than the immune and metabolic tumor status. This most likely explains why the excellent short-term survival of favorable immune and metabolic tumor phenotypes does not result in a superiority regarding long-term survival. Therefore, long-term survival of these advanced tumor patients seems to be determined by other factors than immune and metabolic tumor phenotype. One important parameter in this context is p16 positivity as a surrogate marker for HPV infection in OPSCC. HPV-positive OPSCCs are known for their good radiotherapy response. Additionally, we have shown in a previous study that 
HPV-positivity in OPSCC is associated with high numbers of tumor-infiltrating $\mathrm{CD}^{+}$CTL and a glucose-independent, mitochondrial-rich tumor metabolism. ${ }^{16}$ In our study, however, p16-positive HNSCC only showed a trend toward better survival, which is probably due to the low number of positive cases.

In summary, our study demonstrates a strong connection between the TIME and tumor metabolism, and points out the relevance of this interdependence for therapy response in HNSCC patients. Mechanistically, our study suggests ROSdependent signaling as one potential mediator connecting tumor metabolism and the TIME. Together, these findings point toward future strategies for integration of assessment of immune and metabolic marker combinations in the clinical setting to select patients likely to benefit from CRT.

\section{Acknowledgment}

Survival data were provided by the Tumor Center Regensburg.

\section{Supplemental Data}

Supplemental material for this article can be found at https://doi.org/10.1016/j.ajpath.2017.09.013.

\section{References}

1. Siegel RL, Miller KD, Jemal A: Cancer statistics, 2016. CA Cancer J Clin 2016, 66:7-30

2. Grégoire V, Lefebvre J-L, Licitra L, Felip E; EHNS-ESMO-ESTRO Guidelines Working Group: Squamous cell carcinoma of the head and neck: EHNS-ESMO-ESTRO Clinical Practice Guidelines for diagnosis, treatment and follow-up. Ann Oncol 2010, 21 Suppl 5:v184-v186

3. Balermpas P, Michel Y, Wagenblast J, Seitz O, Weiss C, Rödel F, Rödel C, Fokas E: Tumour-infiltrating lymphocytes predict response to definitive chemoradiotherapy in head and neck cancer. Br J Cancer 2014, 110:501-509

4. Shim BY, Jung J-H, Lee K-M, Kim H-J, Hong SH, Kim SH, Sun DS, Cho H-M: Glucose transporter 1 (GLUT1) of anaerobic glycolysis as predictive and prognostic values in neoadjuvant chemoradiotherapy and laparoscopic surgery for locally advanced rectal cancer. Int $\mathrm{J}$ Colorectal Dis 2013, 28:375-383

5. Fokas E, McKenna WG, Muschel RJ: The impact of tumor microenvironment on cancer treatment and its modulation by direct and indirect antivascular strategies. Cancer Metastasis Rev 2012, 31:823-842

6. Lynam-Lennon N, Maher SG, Maguire A, Phelan J, Muldoon C, Reynolds JV, O'Sullivan J: Altered mitochondrial function and energy metabolism is associated with a radioresistant phenotype in oesophageal adenocarcinoma. PLoS One 2014, 9:e100738

7. Dewhirst MW, Cao Y, Moeller B: Cycling hypoxia and free radicals regulate angiogenesis and radiotherapy response. Nat Rev Cancer 2008, 8:425-437

8. Mims J, Bansal N, Bharadwaj MS, Chen X, Molina AJ, Tsang AW, Furdui CM: Energy metabolism in a matched model of radiation resistance for head and neck squamous cell cancer. Radiat Res 2015, 183:291-304

9. Groussard C, Morel I, Chevanne M, Monnier M, Cillard J, Delamarche A: Free radical scavenging and antioxidant effects of lactate ion: an in vitro study. J Appl Physiol (1985) 2000, 89:169-175
10. Bhatt AN, Chauhan A, Khanna S, Rai Y, Singh S, Soni R, Kalra N, Dwarakanath BS: Transient elevation of glycolysis confers radioresistance by facilitating DNA repair in cells. BMC Cancer 2015, 15:335

11. Senyilmaz D, Teleman AA: Chicken or the egg: Warburg effect and mitochondrial dysfunction. F1000prime Rep 2015, 7:41

12. Curry JM, Tuluc M, Whitaker-Menezes D, Ames JA, Anantharaman A, Butera A, Leiby B, Cognetti DM, Sotgia F, Lisanti MP, MartinezOutschoorn UE: Cancer metabolism, stemness and tumor recurrence: MCT1 and MCT4 are functional biomarkers of metabolic symbiosis in head and neck cancer. Cell Cycle 2013, 12:1371-1384

13. Hirschhaeuser F, Sattler UGA, Mueller-Klieser W: Lactate: a metabolic key player in cancer. Cancer Res 2011, 71:6921-6925

14. Fischer K, Hoffmann P, Voelkl S, Meidenbauer N, Ammer J, Edinger M, Gottfried E, Schwarz S, Rothe G, Hoves S, Renner K, Timischl B, Mackensen A, Kunz-Schughart L, Andreesen R, Krause SW, Kreutz M: Inhibitory effect of tumor cell-derived lactic acid on human T cells. Blood 2007, 109:3812-3819

15. Sattler UGA, Meyer SS, Quennet V, Hoerner C, Knoerzer H, Fabian C, Yaromina A, Zips D, Walenta S, Baumann M, Mueller-Klieser W: Glycolytic metabolism and tumour response to fractionated irradiation. Radiother Oncol 2010, 94:102-109

16. Krupar R, Robold K, Gaag D, Spanier G, Kreutz M, Renner K, Hellerbrand C, Hofstaedter F, Bosserhoff AK: Immunologic and metabolic characteristics of HPV-negative and HPV-positive head and neck squamous cell carcinomas are strikingly different. Virchows Arch 2014, 465:299-312

17. Brenner JC, Graham MP, Kumar B, Saunders LM, Kupfer R, Lyons RH, Bradford CR, Carey TE: Genotyping of 73 UM-SCC head and neck squamous cell carcinoma cell lines. Head Neck 2010, 32:417-426

18. Li S, Yang X, Wang P, Ran X: The effects of GLUT1 on the survival of head and neck squamous cell carcinoma. Cell Physiol Biochem 2013, 32:624-634

19. Shimura T, Noma N, Sano Y, Ochiai Y, Oikawa T, Fukumoto M, Kunugita N: AKT-mediated enhanced aerobic glycolysis causes acquired radioresistance by human tumor cells. Radiother Oncol 2014 , 112:302-307

20. Starska K, Forma E, Jóźwiak P, Bryś M, Lewy-Trenda I, BrzezińskaBłaszczyk E, Krześlak A: Gene and protein expression of glucose transporter 1 and glucose transporter 3 in human laryngeal cancer-the relationship with regulatory hypoxia-inducible factor- $1 \alpha$ expression, tumor invasiveness, and patient prognosis. Tumour Biol 2015, 36:2309-2321

21. Goldkorn T, Balaban N, Shannon M, Chea V, Matsukuma K, Gilchrist D, Wang H, Chan C: $\mathrm{H} 2 \mathrm{O} 2$ acts on cellular membranes to generate ceramide signaling and initiate apoptosis in tracheobronchial epithelial cells. J Cell Sci 1998, 111:3209-3220

22. Reth M: Hydrogen peroxide as second messenger in lymphocyte activation. Nat Immunol 2002, 3:1129-1134

23. Fleury C, Mignotte B, Vayssière J-L: Mitochondrial reactive oxygen species in cell death signaling. Biochimie 2002, 84:131-141

24. Leach JK, Van Tuyle G, Lin PS, Schmidt-Ullrich R, Mikkelsen RB: Ionizing radiation-induced, mitochondria-dependent generation of reactive oxygen/nitrogen. Cancer Res 2001, 61:3894-3901

25. Lennicke C, Rahn J, Lichtenfels R, Wessjohann LA, Seliger B: Hydrogen peroxide - production, fate and role in redox signaling of tumor cells. Cell Commun Signal 2015, 13:39

26. Sun S-Y: N-acetylcysteine, reactive oxygen species and beyond. Cancer Biol Ther 2010, 9:109-110

27. Tong CCL, Kao J, Sikora AG: Recognizing and reversing the immunosuppressive tumor microenvironment of head and neck cancer Immunol Res 2012, 54:266-274

28. Aarstad HJ, Vintermyr OK, Ulvestad E, Aarstad HH, Kross KW Heimdal JH: Peripheral blood monocyte and T-lymphocyte activation levels at diagnosis predict long-term survival in head and neck squamous cell carcinoma patients. APMIS 2015, 123:305-314

29. Anitei M-G, Zeitoun G, Mlecnik B, Marliot F, Haicheur N, Todosi A-M, Kirilovsky A, Lagorce C, Bindea G, Ferariu D, Danciu M, Bruneval P, Scripcariu V, Chevallier J-M, Zinzindohoué F, Berger A, Galon J, 
Pagès F: Prognostic and predictive values of the Immunoscore in patients with rectal cancer. Clin Cancer Res 2014, 20:1891-1899

30. Cho Y, Miyamoto M, Kato K, Fukunaga A, Shichinohe T, Kawarada Y, Hida Y, Oshikiri T, Kurokawa T, Suzuoki M, Nakakubo Y, Hiraoka K, Murakami S, Shinohara T, Itoh T, Okushiba S, Kondo S, Katoh H: CD4+ and CD8+ T cells cooperate to improve prognosis of patients with esophageal squamous cell carcinoma. Cancer Res 2003, 63:1555-1559

31. Huang Y, Ma C, Zhang Q, Ye J, Wang F, Zhang Y, Hunborg P, Varvares MA, Hoft DF, Hsueh EC, Peng G: CD4+ and CD8+ T cells have opposing roles in breast cancer progression and outcome. Oncotarget 2015, 6:17462-17478

32. Maleki S, Schlecht NF, Keller C, Diaz J, Moss J, Prystowsky MB, Macian F, Brandwein-Gensler M: Lymphocytic host response to oral squamous cell carcinoma: an adaptive T-cell response at the tumor interface. Head Neck Pathol 2011, 5:117-122

33. Gooden MJM, de Bock GH, Leffers N, Daemen T, Nijman HW: The prognostic influence of tumour-infiltrating lymphocytes in cancer: a systematic review with meta-analysis. Br J Cancer 2011, 105:93-103

34. Sawayama H, Ishimoto T, Watanabe M, Yoshida N, Baba Y, Sugihara H, Izumi D, Kurashige J, Baba H: High expression of glucose transporter 1 on primary lesions of esophageal squamous cell carcinoma is associated with hematogenous recurrence. Ann Surg Oncol 2014, 21:1756-1762

35. Dang C, Gottschling M, Manning K, O'Currain E, Schneider S, Sterry W, Stockfleth E, Nindl I: Identification of dysregulated genes in cutaneous squamous cell carcinoma. Oncol Rep 2006, 16:513-519

36. Gao S-P, Sun H-F, Jiang H-L, Li L-D, Hu X, Xu X-E, Jin W: Loss of COX5B inhibits proliferation and promotes senescence via mitochondrial dysfunction in breast cancer. Oncotarget 2015, 6:43363-43374

37. Fiaschi T, Marini A, Giannoni E, Taddei ML, Gandellini P, De Donatis A, Lanciotti M, Serni S, Cirri P, Chiarugi P: Reciprocal metabolic reprogramming through lactate shuttle coordinately influences tumor-stroma interplay. Cancer Res 2012, 72:5130-5140

38. Walenta S, Schroeder T, Mueller-Klieser W: Lactate in solid malignant tumors: potential basis of a metabolic classification in clinical oncology. Curr Med Chem 2004, 11:2195-2204

39. Sattler UGA, Mueller-Klieser W: The anti-oxidant capacity of tumour glycolysis. Int J Radiat Biol 2009, 85:963-971
40. Michalek RD, Gerriets VA, Jacobs SR, Macintyre AN, MacIver NJ, Mason EF, Sullivan SA, Nichols AG, Rathmell JC: Cutting edge: distinct glycolytic and lipid oxidative metabolic programs are essential for effector and regulatory CD4+ T cell subsets. J Immunol 2011, 186:3299-3303

41. Tominaga H, Kodama S, Matsuda N, Suzuki K, Watanabe M: Involvement of reactive oxygen species (ROS) in the induction of genetic instability by radiation. J Radiat Res 2004, 45:181-188

42. Cantoni O, Murray D, Meyn RE: Effect of 3-aminobenzamide on DNA strand-break rejoining and cytotoxicity in $\mathrm{CHO}$ cells treated with hydrogen peroxide. Biochim Biophys Acta 1986, 867:135-143

43. Cook JA, Gius D, Wink DA, Krishna MC, Russo A, Mitchell JB: Oxidative stress, redox, and the tumor microenvironment. Semin Radiat Oncol 2004, 14:259-266

44. Urano M, Kuroda M, Reynolds R, Oberley TD, St Clair DK: Expression of manganese superoxide dismutase reduces tumor control radiation dose: gene-radiotherapy. Cancer Res 1995, 55:2490-2493

45. Shutt DC, O'Dorisio MS, Aykin-Burns N, Spitz DR: 2-Deoxy-Dglucose induces oxidative stress and cell killing in human neuroblastoma cells. Cancer Biol Ther 2010, 9:853-861

46. Coleman MC, Asbury CR, Daniels D, Du J, Aykin-Burns N, Smith BJ, Li L, Spitz DR, Cullen JJ: 2-Deoxy-d-glucose causes cytotoxicity, oxidative stress, and radiosensitization in pancreatic cancer. Free Radic Biol Med 2008, 44:322-331

47. Kalia VK, Prabhakara S, Narayanan V: Modulation of cellular radiation responses by 2-deoxy-D-glucose and other glycolytic inhibitors: implications for cancer therapy. J Cancer Res Ther 2009, 5 Suppl 1: S57-S60

48. Hara-Chikuma M, Chikuma S, Sugiyama Y, Kabashima K, Verkman AS, Inoue S, Miyachi Y: Chemokine-dependent T cell migration requires aquaporin-3-mediated hydrogen peroxide uptake. J Exp Med 2012, 209:1743-1752

49. Ashcraft KA, Boss M-K, Tovmasyan A, Roy Choudhury K, Fontanella AN, Young KH, Palmer GM, Birer SR, Landon CD, Park W, Das SK, Weitner T, Sheng H, Warner DS, Brizel DM, Spasojevic I, Batinic-Haberle I, Dewhirst MW: Novel manganeseporphyrin superoxide dismutase-mimetic widens the therapeutic margin in a preclinical head and neck cancer model. Int J Radiat Oncol 2015, 93:892-900 